\title{
Impact of garlic on cardio protective potential of ramipril on ischemia-reperfusion induced myocardial dysfunction in rats
}

\begin{abstract}
Garlic (Allium sativum) is one of the most popular herbs used worldwide to reduce various risk factors associated with cardiovascular diseases. Angiotensin converting enzyme inhibitor (ACE) such as Ramipril (RAM) has been proved to work under conditions of myocardial damage, however, there is no report on elucidating the impact of garlic on its cardioprotective potential. In the current study, role of garlic homogenate $(\mathrm{GH}, 250 \mathrm{mg} / \mathrm{kg}$ ) along with RAM $(1 \mathrm{mg} / \mathrm{kg})$ was investigated in ischemia-reperfusion induced myocardial damage in rats. Sprague dawley rats $(200-250 \mathrm{~g})$ were divided into 4 group $(\mathrm{n}=6)$. Rats were given treatment for 10 days with ramipril and 30days with Garlic in their respective groups. At the end of treatment, heart was isolated under anesthesia and mounted at modified Langendorff's apparatus and myocardial damage was induced by no-flow global ischemia and reperfusion. Percentage recovery in terms of developed tension and heart rate during post-ischemia, $\mathrm{LDH}$ and $\mathrm{CK}-\mathrm{MB}$ activities in perfusate and heart tissue homogenate (HTH), superoxide dismutase (SOD) and Catalase HTH and histopathological scores were evaluated. Administration of GH and RAM resulted in elevation of LDH and CKMB activities in HTH with a simultaneous fall in perfusate. Prophylactic administration of RAM and GH demonstrated good recovery in developed tension and heart rate compared to ischemic control. Moreover, SOD and Catalase activities were also increased in treated groups. Corroborating with the biochemical results, histological scores were diminished in all treated groups. To conclude, concurrent administration of RAM with GH showed better cardioprotective potential than their individual treatment.
\end{abstract}

Keywords: garlic homogenate, interaction, ischemia reperfusion, ramipril
Volume 5 Issue 3 - 2017

\author{
Suresh Kumar V,' Mohammed Basheeruddin \\ Asdaq $q^{1,2}$ \\ 'Department of Pharmacology, Krupanidhi College of Pharmacy, \\ India \\ 2Department of Pharmacology and Therapeutics, Al-Maarefa \\ Colleges for Science and Technology, Saudi Arabia
}

\begin{abstract}
Correspondence: Syed Mohammed Basheeruddin Asdaq Associate Professor, Department of Pharmacology, College of Pharmacy, Al-Maarefa College for Science and Technology, PO Box: 71666, Dariyah, Riyadh-I 1597, Saudi Arabia, Tel 009664903555-3399, Email drznaturally@gmail.com
\end{abstract}

Received: November 02, 2016 | Published: January 25, 2017
Abbreviations: IRI, ischemia-reperfusion injury; RAM, ramipril; $\mathrm{GH}$, garlic homogenate; $\mathrm{SOD}$, superoxide dismutase; CAT, catalase; TBARS, thiobarbituric acid reactive species; HTH, heart tissue homogenate; LDH, lactate dehydrogenase; CK-MB, creatine kinase-MB; MI, myocardial infarction

\section{Introduction}

Ischemic heart diseases (IHD), especially acute myocardial infarction (MI), remain the leading cause of death in both developed and developing countries as seen over the past quarter century and ischemic-reperfusion injury (IRI) is a major pathological factor in the natural course of IHD. ${ }^{1}$ Free radicals and reactive oxygen species have been implicated in the pathogenesis of a variety of diseases such as atherosclerosis, cancer and myocardial ischemia. ${ }^{2}$ Myocardial ischaemia causes pathological changes and tissue injury. However, reperfusion itself may have deleterious effects on the ischaemic myocardium by giving rise to arrhythmias, resulting in prolonged left ventricular dysfunction ("myocardial stunning") and limiting the amount of potentially salvageable myocardium by the conversion of reversibly injured myocytes to irreversibly injured cells. ${ }^{2,3}$ Oxidative stress, due to overwhelming generation of oxygen free radicals (OFR), with concomitant depletion of certain key endogenous antioxidant compounds, such as superoxide dismutase (SOD) and catalase (CAT) plays an important role in the IRI of heart. ${ }^{4}$ Increased appreciation of the occurrence of free radicals, their injury potential and role in the pathogenesis of several disease states demand quantitative methods which are diagnostic of the process. However, it is difficult to quantify free radicals because of their short half-life and reactive nature.
Therefore, indirect methods such as lipid peroxidation and Total protein estimation are preferred. ${ }^{5}$

Angiotensin-converting enzyme inhibitors (ACE) are known to improve left ventricular (LV) function, reduce the incidence of myocardial infarction (MI) and stroke, prolong survival in patients with congestive heart failure (CHF) and benefit all forms of cardiovascular disease. ${ }^{6}$ The effects of ACE have been attributed not only to blockade of angiotensin II (Ang II) formation, but also to inhibition of kinin degradation and increased production of nitric oxide and prostacyclin. ${ }^{7,8}$ The renin-angiotensin system is present both in the circulation and in different tissues. Both the cardiac renin-angiotensin system and $\mathrm{ACE}$, which catalyses the final step in the enzymatic cascade of renin-angiotensin system, appear to be activated in various conditions including myocardial ischaemia. ${ }^{9-11}$ Recent studies have shown that under appropriate experimental conditions of myocardial ischaemia reperfusion, ACE inhibitors can reduce the incidence and duration of ventricular arrhythmias, improve contractile dysfunction and limit infarct size. ${ }^{10}$ In addition to their antihypertensive effect, ACE inhibitors have anti-inflammatory properties, improve nitric oxide (NO) bioavailability in human vasculature, and modify cardiovascular risk by interfering directly into the mechanisms of atherogenesis. ${ }^{12}$ The underlying mechanism of these cardioprotective effects is not fully understood.

The health benefits acquired by garlic consumption have been recognized since early period of history. ${ }^{13}$ The pharmacological activities (i.e. antihypertensive, lipid-lowering, antiatherogenic, antitumorigenic, fibrinolytic, antioxidant, anticarcinogenic, immunomodulatory, antimicrobial, antihepatotoxic, hypoglycemic 
and other effects) of various garlic constituents ${ }^{14}$ and garlic preparations ${ }^{15}$ have been confirmed in numerous epidemiological human studies, preclinical animal studies and extensive in vitro research. ${ }^{15,16}$ The general belief that over-the-counter products are safe and lack adverse effects contributes to constantly rising simultaneous consumption of dietary supplements with or even instead of prescribed drugs. ${ }^{17}$ Therefore, the present study was designed to investigate the effects of garlic administration on cardioprotective effect of ramipril: on endogenous antioxidant status; and on biochemical estimation of cardiac markers arising out of ischemic-reperfusion injury of heart.

\section{Materials and methods}

\section{Study design}

Institution Animal Ethics Committee approved the experimental protocol. Male Sprague Dawley rats weighing 200-250g were housed at $25^{\circ} \pm 5{ }^{\circ} \mathrm{C}$ in a well-ventilated animal house under 12-h light:12-h dark cycle, where they had free access to food (std pellet diet), water and libitum. The animals were maintained under standard conditions in an animal house as per the guidelines of Committee for the Purpose of Control and Supervision on Experiments on Animals (CPCSEA). The Institutional ethical committee approved the experimental protocol (KCP/IAEC-27/2009-10).

\section{Materials used}

Ramipril was purchased from Micro Labs Company Ltd. LDH and CK-MB Kits were purchased from Crest Biosystems. All other chemicals and drugs used in the study were procured from standard companies and they were of analytical grade.

\section{Preparation of garlic homogenate}

Garlic (Allium sativum, family: Lilliaceae) bulbs were purchased from the local vegetable market. The cloves were peeled, sliced and ground into a paste and suspended in distilled water. The garlic homogenate (GH) were prepared, $0.1 \mathrm{gm} / \mathrm{ml}$, corresponding to $250 \mathrm{mg} /$ $\mathrm{kg}$ body weight of animal. ${ }^{19}$ The Aqueous GH was administered by oral gavage everyday within $30 \mathrm{~min}$ of preparation.

\section{Experimental protocol}

Rats were randomly allocated into four groups and treated Orally in which Group-I was kept as control (saline), Group-II was treated with GH $(250 \mathrm{mg} / \mathrm{kg})$ (19) for 30days, Group-III was treated with RAM (1mg/kg)(20) for last 10days and Group- IV was administered with $\mathrm{GH}(250 \mathrm{mg} / \mathrm{kg})$ for 30days and RAM $(1 \mathrm{mg} / \mathrm{kg})$ for last 10days.

\section{Biochemical parameters}

Cardiac markers such as CKMB \& LDH were estimated by a method described by Asdaq \& Inamdar. ${ }^{19}$ Thiobarbituric acid reactive substances (TBARS) ${ }^{21,22}$ was measured as a marker of lipid peroxidation and endogenous antioxidants, e.g., superoxide dismutase (SOD) (23) and catalase (24), were also estimated.

\section{Measurement of cardiovascular hemodynamics}

After $15 \mathrm{~min}$ equilibration, Heart rate, Developed Tension, contractile force were measured by displacement transducer and recorded on a grass electromechanical recorder.

\section{Perfusion of isolated rat heart}

Animal models of myocardial ischemia and reperfusion was done according to the method previously described with slight modifications. ${ }^{25}$ At the end of treatment, heart will be excised from deeply anesthetized rat $(35 \mathrm{mg} / \mathrm{kg}$ sodium pentobarbitone, i.p) and perfused with Krebs-Henseleit solution (in gm/lit): NaCl- 6.9, $\mathrm{KCl}$ $-0.35, \mathrm{KH} 2 \mathrm{PO} 4-1.2, \mathrm{CaCl} 2-0.28, \mathrm{MgSO} 4-0.133, \mathrm{NaHCO} 3-2.1$ and glucose-1gm/lit. Within $2 \mathrm{~min}$ after thoracotomy the heart was removed, mounted on the experimental setup and perfused retrogradely at $5 \mathrm{ml} / \mathrm{min}$ (peristaltic pump) by one-way circulation using modified Langendorff's preparation through the aorta with Krebs-Henseleit solution, which was kept at $37^{\circ} \mathrm{C}$ and aerated with $95 \% \mathrm{O} 2+5 \%$ $\mathrm{CO} 2$ to maintain normal pH, pO2 and $\mathrm{pCO} 2$ levels. Measurement of contractile force was done with displacement transducer and recorded on a grass electromechanical recorder.

\section{Myocardial ischemia and reperfusion}

After $15 \mathrm{~min}$ equilibration, records were taken for a control period of 15 minutes, and then followed by 15 minutes global ischemia and reperfusion. The normal flow rate $(5 \mathrm{ml} / \mathrm{min})$ was then restored and perfusion was continued for a further $30 \mathrm{~min}$ after reperfusion. Recovery in terms of isotropic and chronotropic effect were studied and the extent of cardioprotection due to prophylactic therapies were evaluated by measuring the developed tension. Throughout the experiment, a thermo-regulated chamber kept the heart at $37^{\circ} \mathrm{C}$ to avoid hypothermia-induced cardio-protection. None of the experiments lasted more than $2 \mathrm{~h}$, during which time the experimental preparation was stable.

\section{Histopathological studies}

Microscopic slides of myocardium were prepared for histopathological studies from the hearts of remaining four animals. The myocardial damage was determined by giving scores depending on the intensity as follows; ${ }^{26}$ no changes- score 00 ; mild- score 01 (focal myocytes damage or small multifocal degeneration with slight degree of inflammatory process); moderate- score 02 (extensive myofibrillar degeneration and/or diffuse inflammatory process); marked- score 03 (necrosis with diffuse inflammatory process).

\section{Statistical analysis}

The statistical significance will be assessed using one-way analysis of variance (ANOVA) followed by Bonferoni comparison test. The values are expressed as mean \pm SEM and $p<0.05$ will be considered significant.

\section{Results}

\section{Effects on SOD, catalase and TBARS}

There was significant $(p<0.05)$ increase in myocardial TBARS in the group ISC when compared to the GH. Significant decrease in the level of myocardial TBARS was observed in GH+RAM treated groups $(\mathrm{p}<0.001)$ in comparison to the GH group (Table 1). In ISC group, there was a significant reduction in myocardial SOD activity when compared to GH group. Significant $(p<0.001)$ increase in myocardial SOD activities was observed in groups GH and RAM group when compared to ISC (Table 1). In the ISC group there was a significant $(p<0.001)$ decrease in myocardial catalase activity compared to GH. In the RAM groups there was no significant increase in the level of myocardial catalase activities compared to ISC. However, there is significant $(\mathrm{p}<0.001)$ increase in myocardial catalase activity was seen in combination of GH and RAM treated group (Table 1).

\section{Effects on CKMB and LDH}

In the ISC group there was a significant $(\mathrm{p}<0.001)$ decrease in LDH and CKMB levels in perfusate when compared with HTH showing protective action of GH. There was also significant $(\mathrm{p}<0.001)$ 
recovery from global ischemia in groups treated with $\mathrm{GH}$ alone or with RAM (Table 2).

\section{Effects on developed tension and heart rate}

Pretreatment of RAM significantly $(\mathrm{p}<0.001)$ imparts the recovery to ischemic heart in terms of developed tension and heart rate. There was also significant $(\mathrm{p}<0.001)$ recovery from global ischemia in groups treated with $\mathrm{GH}$ alone or with RAM (Table 3).

\section{Effect on histological parameters}

The microscopic section of heart muscles of animals subjected to ischemia-reperfusion showed intervening fibrovascular septae (Figures 1 \& 2). Administration of RAM caused reduction in intervening fibrovascular septae and the cardiac muscle appears to be within normal limits (Figure 3). The combined therapy of GH with ramipril was more cardioprotective with substantially reduced coagulative necrosis (Figure 4).

Table I Effects on SOD, catalase and TBARS in heart tissue homogenate against Ischemia reperfusion induced damage

\begin{tabular}{|c|c|c|c|}
\hline \multicolumn{4}{|c|}{ Heart Tissue Homogenate } \\
\hline Treatment & SOD (unit/mg Protein) & Catalase (unit/mg Protein) & TBARS (unit/mg Protein) \\
\hline ISC & $0.93 \pm 0.52$ & $3.03 \pm 0.38$ & $47.83 \pm 0.27$ \\
\hline $\mathrm{GH}$ & $3.39 \pm 0.48$ & $10.73 \pm 0.76$ & $26.1 \pm 3.60$ \\
\hline RAM & $2.42 \pm 0.69$ & $8.95 \pm 0.40$ & $22.83 \pm 2.06$ \\
\hline $\mathrm{RAM}+\mathrm{GH}$ & $9.57 \pm 1.01 * * * *^{00}{ }^{\circ} \cap \Omega($ & $|4.5| \pm \mid .85 * \cap \Omega \Omega^{\circ}$ & $|6.4 \pm 0.3|^{* * \circ \circ} \AA \circledast \cap$ \\
\hline
\end{tabular}

Table 2 Effects on LDH and CKMB level in perfusate and heart tissue homogenate against Ischemia reperfusion induced damage

\begin{tabular}{|c|c|c|c|c|}
\hline \multirow{2}{*}{ Treatments } & \multicolumn{2}{|l|}{ CK-MB Activity } & \multicolumn{2}{|l|}{ LDH Activity } \\
\hline & Perfusate (unit/lit) & HTH (unit/gm) & Perfusate (unit/lit) & HTH (unit/gm) \\
\hline ISC & $144 \pm 22.1$ & $42.4 \pm 4.3$ & $142 \pm \mid 4.5$ & $31.5 \pm 7.8$ \\
\hline $\mathrm{GH}$ & $115 \pm 3.6$ & $18.1 \pm 1 \mid .6$ & $117 \pm 27.9$ & $4.0 \pm 1.2 \circledast \circledast \circledast$ \\
\hline RAM & $442 \pm 27.2^{* * *}$ & $83.3 \pm 29.1 \circledast \Re$ & $163 \pm 8.6$ & $2.5 \pm 0.4 \AA R \circledast$ \\
\hline $\mathrm{RAM}+\mathrm{GH}$ & $473 \pm 27.0 * * * \cap \AA \Omega$ & $441.4 \pm 30.9 * * * \Omega \Omega \Omega^{\circ 00}$ & $395 \pm 27.2 * * * \cap R \Omega^{000}$ & $8.8 \pm 6.0 \AA R \Omega^{\circ 00 * *}$ \\
\hline
\end{tabular}

Table 3 Percentage recovery in terms of heart rate and developed tension against Ischemia reperfusion induced damage

\begin{tabular}{lll}
\hline Treatments & \% Recovery of Heart Rate & \% Recovery of Developed Tension \\
\hline ISC & $48.2 \pm I .8$ & $54.3 \pm 2.8$ \\
GH & $65.6 \pm 3.4 *$ & $75.0 \pm 1.7^{* *}$ \\
RAM & $57.6 \pm 4.3$ & $63.3 \pm 3.2$ \\
RAM + GH & $\left.82.6 \pm 1.4^{* * *}\right)^{\circ \circ}$ & $85.6 \pm I .7^{* * *(R \cap}{ }^{\circ}$ \\
\hline
\end{tabular}

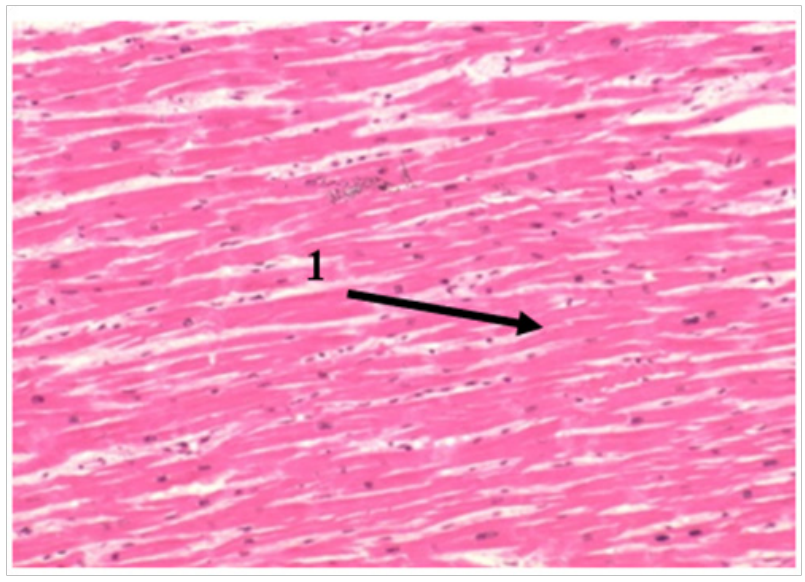

Figure I (H\&E, 400X) Heart tissue of normal. Normal texture of cell.

\section{Discussion}

The present study was designed to evaluate the pharmacological efficacy of Aqueous GH and interaction with RAM against ischemic reperfusion injury (IRI) in normal rats. The results indicated that an increase in endogenous antioxidant activities in HTH is indication for structural integrity and protection to the myocardium that were achieved by prior administration of GH alone and along with RAM. In the current study it is reported that chronic oral administration of raw garlic homogenate (30days) caused a significant increase in basal SOD and catalase activities of HTH, which was associated with a concomitant decrease in basal lipid peroxidation measured as TBARS. Ischemic reperfusion injury (IRI) is associated with increased oxidative damage, as evidenced by increase in myocardial TBARS and depletion of myocardial endogenous antioxidants such as SOD, catalase. Similar observations were made earlier by different other studies, using similar models. ${ }^{27-29}$ Myocardial adaptation against oxidative stress is mediated through augmentation of a number of cellular antioxidants, such as SOD, catalase, glutathione peroxidase, glutathione. ${ }^{23}$ There is increase in the heart rate in GH treated group and decrease in heart rate in RAM treated group, in combination there was significant increase in heart rate when compared with ischemic control (ISC) at $\mathrm{P}<0.05$, percentage recovery of the heart rate was also calculated. The developed tension was significantly increased in GH and RAM treated group and in combination with RAM when compared with ISC.

The duration and severity of myocardial ischemia are clearly the most important determinants of irreversible myocardial damage, and the potentially harmful consequences of myocardial reperfusion causing leakage of Cardiac biomarkers such as CK-MB, LDH and Troponin. Oxidative damage to the myocardial membrane due to ischemic reperfusion injury leads to leakage of endogenous cardiac biomarkers into the circulation and thereby decreasing the intracellular 
enzyme levels. GH may provides protection to myocardium by scavenging oxidative free radicals and thereby diminishing the permeability of these endogenous biomarkers to extra cardiac regions (perfusate). Cardiac markers like CK-MB and LDH were found to decline in perfusate when compared with HTH showing protective action may be due to the presence of high concentration of saponin ${ }^{30}$ in garlic which have membrane stabilizing property against ischemia reperfusion induced oxidative damage in treated groups when compared with control group.

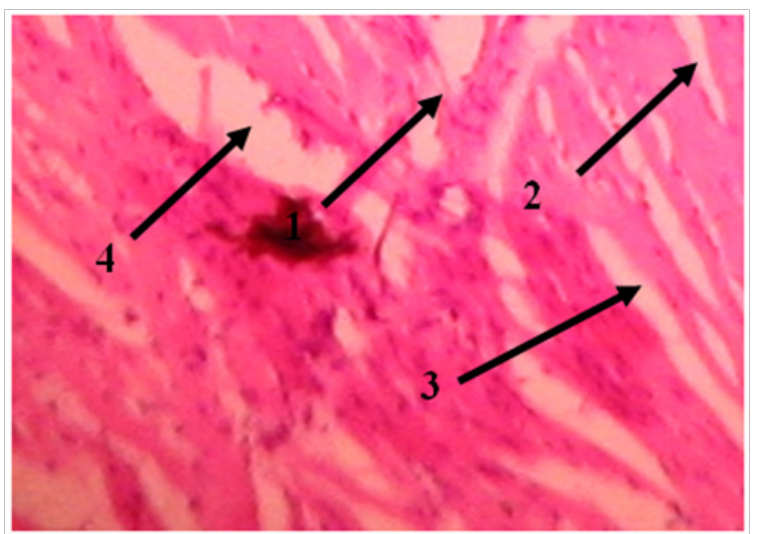

Figure 2 (H\&E, 400X) ischemic control.

I. Myocardial Oedema; 2. Separation of Fibres; 3. Loss of Striation; 4. Necrosis

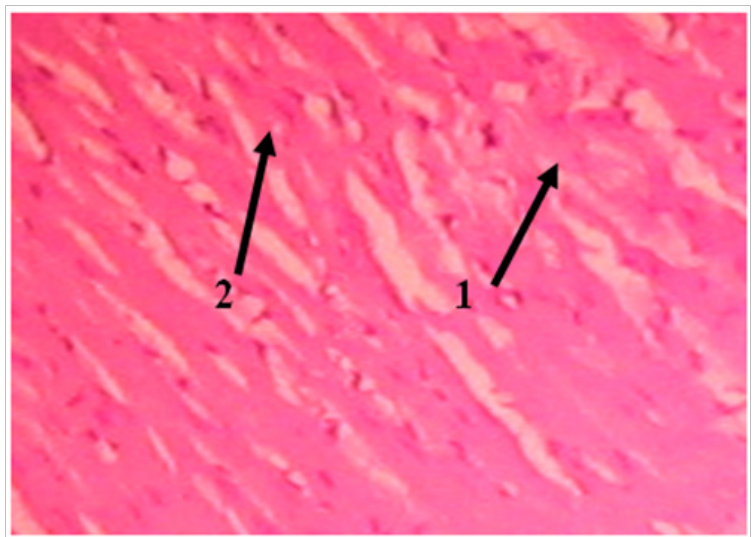

Figure 3 (H\&E, 400X) RAM ( I mg/kg).

I. Size of the cardiocytes decreased.

2. Intracellular space increased.

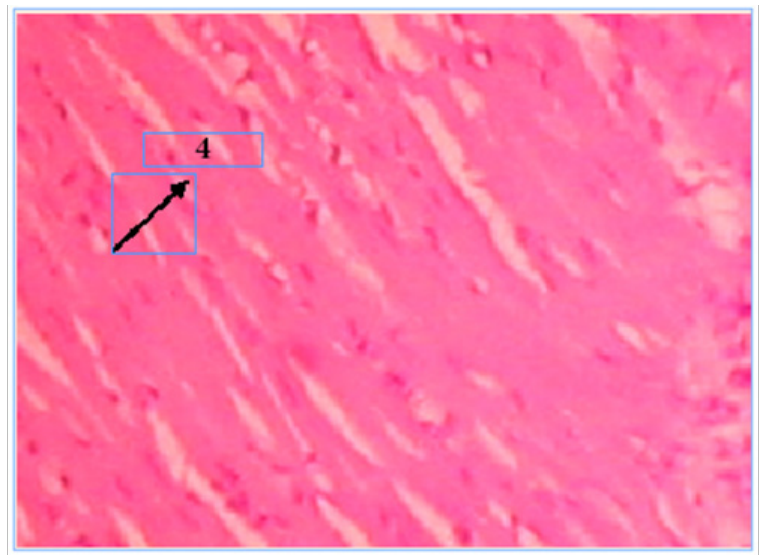

Figure 4 (H\&E, 400X) RAM(I mg/kg) $+\mathrm{GH}(250 \mathrm{mg} / \mathrm{kg})$.

I. Normal structure of myocyte with slight edema.
Garlic is also reported to inhibit the pathogenesis of cardiovascular disease and to prevent cancer and other chronic diseases associated with aging. ${ }^{31}$ Over the last one-quarter century the role of garlic in treating cardiovascular disease has received much attention. The majority of garlic $(65 \%)$ is water, and the bulk of the dry weight is composed of fructose-containing carbohydrates, followed by sulfur compounds, protein, fiber, and free amino acids. ${ }^{32}$ It also contains high levels of saponins, phosphorus, potassium, sulfur, zinc, moderate levels of selenium and Vitamins A and C, and low levels of calcium, magnesium, sodium, iron, manganese, and B-complex vitamins; garlic also has a high phenolic content. ${ }^{30} \mathrm{~A}$ majority of the compounds present in garlic are water-soluble $(97 \%)$ with small amounts of oilsoluble compounds also present $(0.15-0.7 \%)$.

ACE may also be involved in the process of tissue repair. It was reported that ACE expression in vascular smooth muscle cells was induced after intimal injury. ${ }^{33}$ In addition, ACE inhibitors are able to prevent myointimal proliferation. ${ }^{34}$ Thus, bradykinin potentiating may also play a role in the anti-proliferative action of ACE inhibitors. ${ }^{35}$ Over-expression of ACE was reported to be involved in the process of cardiac remodeling following MI. ${ }^{36,37}$ Increased ACE synthesis may lead to markedly enhanced angiotensin II formation. Angiotensin II stimulated growth of myocytes and fibroblast was suggested as a basis for compensatory ventricular remodeling. ${ }^{37}$ In cardiac fibroblasts, elevated angiotensin II levels were shown to increase both mRNA expression and protein synthesis of type-I collagen. ${ }^{38}$ Therefore, high angiotensin II concentrations in the scar tissue of infarcted hearts might activate fibroblast growth and collagen synthesis.

In ramipril-pretreated rats, tissue blood flow significantly increased after coronary artery ligation. ${ }^{39,40}$ In addition, infarct size evaluated 3 weeks after MI was also reduced by ramipril treatment in comparison with that of placebo-treated rats. ACE inhibition with a dose $(1 \mathrm{mg} / \mathrm{kg} /$ day) of ramipril doubled lifespan to 30months in spontaneously hypertensive rats and a dose ( $10 \mathrm{microgram} / \mathrm{kg} / \mathrm{day})$ of ramipril prolonged lifespan from 15 to 18 months. ${ }^{41,42}$

\section{Conclusions}

Prior administration of RAM protects myocardium from ischemiareperfusion induced cardiac dysfunction and metabolic derangement in animal models. Concurrent administration of RAM with GH exhibited greater protection to myocardium. However, further comprehensive studies have to be carried out before its clinical application.

\section{Acknowledgements}

The authors are thankful to the management of Krupanidhi College of Pharmacy, Bangalore, India for providing necessary facilities to carry out part of this research work.

\section{Conflicts of interest}

Author declares there are no conflicts of interest.

\section{Funding}

None.

\section{References}

1. Hansen PR. Myocardial reperfusion injury: experimental evidence and clinical relevance. European Heart Journal. 1995;16(6):734-740.

2. Halliwell B, Gutteridge JMC. Free Radicals in Biology and Medicine. 2nd edn. UK, Oxford: Clarendon Press; 1991. 
3. Forman MB, Puett DW, Virmani R. Endothelial and myocardial injury during ischemia and reperfusion: pathogenesis and therapeutic implications. I Am Coll Cardiol. 1989;13(2):450-499.

4. Ferrari R, Ceconi C, Curello S, et al. The occurrence of oxidative stress during reperfusion in experimental animals and men. Cardiovascular Drugs and Therapeutics. 1991;5(2):277-288.

5. Halliwell B, Gutteridge JMC. Role of free radicals and catalytic metal ions in human disease: an overview. Meth Enzymol. 1990;186:1-85.

6. Yusuf S, Sleight P, Pogue J, et al. The Heart Outcomes Prevention Evaluation Study Investigators. Effects of an angiotensin-converting enzyme inhibitor, ramipril, on cardiovascular events in high-risk patients. N Engl J Med. 2000;342(3):145-153.

7. Blais CJ, Fortin D, Rouleau JL, et al. Protective effect of omapatrilat, a vasopeptidase inhibitor, on the metabolism of bradykinin in normal and failing human hearts. J Pharmacol Exp Ther. 2000;295(5):621-626.

8. Yang XP, Liu YH, Mehta D, et al. Diminished cardioprotective response to inhibition of angiotensinconverting enzyme and angiotensin II type 1 receptor in $\mathrm{B} 2$ kinin receptor gene knockout mice. Circ Res. 2001;88(10):1072-1079.

9. Johnston CI. Renin-angiotensin system: a dual tissue and hormonal system for cardiovascular control. J Hypertens. 1992;10(7):S13-S26.

10. Wijngaarden J, Tio A, Van Gilst WH, et al. Basic pharmacology of ACEinhibitors with respect to ischemic heart disease:prostaglandins and bradykinin. Eur Heart J. 1990;1(Suppl B):84-93.

11. Struthers AD. The clinical pharmacology of angiotensin converting enzyme inhibitors in chronic heart failure. Pharmac Ther. 1992;53(2):187-197.

12. Watanabe T, Barker TA, Berk BC. Angiotensin II and the endothelium: diverse signals and effects. Hypertension. 2005;45(2):163-169.

13. Rivlin RS. Historical perspective on the use of garlic. J Nutr. 2001;131(3S):951S-954S

14. Agarwal KC. Therapeutic actions of garlic constituents. Med Res Rev. 1996;16(1):111-124.

15. Ejaz S, Woong LC, Ejaz A. Extract of garlic (Allium Sativum) in cancer chemoprevention. Exp Oncol. 2003;25:93-97.

16. Yeh YY, Liu L. Cholesterol-lowering effect of garlic extracts and organosulfur compounds: human and animal studies. J Nutr. 2001;131(3S):989S-993S

17. Amagase H. Clarifying the real bioactive constituents of garlic. J Nutr. 2006;136(3Suppl):716S-725S.

18. Asdaq SM, Inamdar MN. The potential for interaction of hydrochlorothiazide with garlic in rats. Chem Biol Interact. 2009;181(3):472-479.

19. Asdaq SM, Inamdar MN, Asad M. Pharmacodynamic interaction of Garlic with Proponalol in Ischemic-reperfusion induced Myocardial damage. Pak J Pharm Sci. 2010;23(1):42-47.

20. Zimmermann R, Kastens J, Linz W, et al. Effect of long-term ACE inhibition on myocardial tissue in hypertensive stroke-prone rats. Journal of Molecular Cell Cardiology. 1999;31(8):1447-1456.

21. Wolf RE, Graeber GM, Burge JR, et al. Evaluation of serum creatine kinase and lactate dehyrogenase in experimental myocardial infarction, atriotomies, and thoracotomies. Ann Thorac Surg. 1986;41(4):378-386.

22. Sedlakova E, Racz O, Lovasova E, et al. Markers of oxidative stress in acute myocardial infarction treated by percutaneous coronary intervention. Cent Eur J Med. 2009;4(1):26-31.
23. Singh PN, Athar MS. Simplified calculation of mean QRS vector (mean electrical axis of heart) of electrocardiogram. Indian J Physiol Pharmacol. 2003;47(2):212-216.

24. Lowry CH, Rose Borough NI, Farr AL, et al. Protein measurement with folin phenol reagent. J Biol Chem. 1951;193(1):265-275.

25. Walter MF, Jacob RF, Jeffers B, et al. Serum levels of thiobarbituric acid reactive substances predict cardiovascular events in patients with stable coronary artery disease. J Am Coll Cardiol. 2004;44(10):1996-2002.

26. Karthikeyan K, SaralaBai BR, Devaraj N. Cardioprotective effect of grape seed proanthocyanidins on isoproterenol-induced myocardial injury in rats. Int J Cardiol. 2007;115(3):326-333.

27. Asdaq SM, Inamdar MN. Pharmacodynamic interaction of garlic with hydrochlorothiazide in rats. Indian $J$ Physiol Pharmacol. 2009;53(2):127-136.

28. Maulik M, Maulik SK, Kumari R. Importance of dosage and timing of magnesium administration: A study on the isolated ischemic-reperfused heart. Magnesium Research. 1999;12(1):37-42.

29. Sun JZ, Tang XL, Park SW, et al. Evidence for an essential role of reactive oxygen species in the genesis of late preconditioning against myocardial stunning in conscious pig. Journal of Clin Invest. 1996;97(2):562-576.

30. Vinson JA, Su X, Zubik L, et al. Phenol antioxidant quantity and qualityin foods: fruits. J Agric Food Chem. 2001;49(11):5315-5321.

31. Rahman K. Garlic and aging: new insights into an old remedy. Ageing Res Rev. 2003;2(1): 39-56.

32. Lawson LD. The composition and chemistry of garlic cloves and processed garlic. In: Koch HP \& Lawson LD, Editors. Garlic the science and therapeutic application of Allium sativum L and related species. USA, Baltimore: Williams \& Wilkins; 1996.p.37-107.

33. Rakugi H, Kim DK, Krieger JE, et al. Induction of angiotensin converting enzyme in the neointima after vascular injury. J Clin Invest. 1994;93(3):339-346.

34. Powell J, Clozel J, Müller R, et al. Inhibitors of angiotensin-converting enzyme preventmyointimal proliferation after vascular injury. Science. 1989;245(4914):186-188.

35. Farhy RD, Ho KL, Carretero OA, et al. Kinins mediate the antiproliferative effect of ramipril in rat carotid artery. Biochem Biophys Res Commun. 1992;182(1):283-238.

36. Falkenhahn M, Franke F, Bohle RM, et al. Cellular distribution of angiotensinconverting enzyme after myocardial infarction.Hypertension. 1995;25(2):219-226.

37. Weber KT, Brilla CG, Janicki JS. Myocardial fibrosis:functional significance and regulatory factors. Cardiovasc Res. 1993;27(3):341348

38. Zhou G, Kandala JC, Tyagi SC, et al. Effects of angiotensin II and aldosterone on collagen gene expression and protein turnover in cardiac fibroblasts.Mol cell Biochem. 1996;154(2):171-178.

39. Stauss HM, Zhu YC, Redlich T, et al. ACE inhibitors in infarct-induced heart failure: bradykinin versus angiotensin II. J Cardiovasc Risk. 1994;1(3):255-262.

40. Zhu YC, Stauss HM, Bao G, et al. Role of bradykinin in the antihypertensive and cardioprotective actions of converting enzyme inhibitors. J Physiol Pharmacol. 1995;73(7):827-831.

41. Linz W, Jessen T, Becker RH, et al.Long-term ACE inhibition doubles lifespan of hypertensive rats. Circulation. 1997;96(9):3164-3072. 